\title{
Expression of cluster of differentiation 34 and vascular endothelial growth factor in breast cancer, and their prognostic significance
}

\author{
ZHANHONG CHEN ${ }^{*}$, SHENHUA XU* , WEIZHEN XU, JIAN HUANG, \\ GU ZHANG, LEI LEI, XIYING SHAO and XIAOJIA WANG
}

\begin{abstract}
Key Laboratory of Technology Research on the Diagnosis and Treatment of Thoracic Cancer, Zhejiang Cancer Hospital, Hangzhou, Zhejiang 310022, P.R. China
\end{abstract}

Received July 4, 2014; Accepted March 6, 2015

DOI: $10.3892 / \mathrm{ol} .2015 .3348$

\begin{abstract}
The aim of the present study was to investigate the immunohistochemical expression of cluster of differentiation (CD) 34 and vascular endothelial growth factor (VEGF) in breast cancer tissue, and their prognostic significance. High CD34 expression levels (microvessel density, $>15 / \mathrm{HPF}$ ) were identified in $27.3 \%$ (12/44) of cases, exhibiting no significant correlation with the clinicopathological characteristics of the patients. However, Kaplan-Meier analysis demonstrated that the survival time of patients with high CD34 expression was significantly shorter than that of patients with low CD34 expression (50.0 vs. $90.6 \%$; $\mathrm{P}=0.003)$. Samples with high VEGF expression levels (++ or +++) accounted for $63.6 \%$ (28/44) of the total number of cases. High VEGF expression was significantly prevalent in patients aged $\geq 50$ years compared with patients aged $<50$ years ( $\leq 78.6$ vs. $37.5 \%$; $\mathrm{P}=0.006)$. Furthermore, all patients with vascular invasion exhibited high VEGF expression levels; thus, patients with vascular invasion presented with significantly higher VEGF expression rates compared with patients with no vascular invasion (100.0 vs. 55.6\%; $\mathrm{P}=0.018$ ). However, Kaplan-Meier analysis demonstrated that high VEGF expression was not correlated with the overall survival of the patients $(\mathrm{P}=0.366)$. By contrast, Cox multivariate analysis identified that clinical stage, triple-negative subtype and age were independent prognostic factors for patients with breast cancer $(\mathrm{P}=0.005, \mathrm{P}=0.006$ and $\mathrm{P}=0.032$, respectively), and that $\mathrm{CD} 34$ expression was a potential independent prognostic factor $(\mathrm{P}=0.055)$. Therefore, the present study determined that
\end{abstract}

Correspondence to: Dr Xiaojia Wang, Key Laboratory of Technology Research on the Diagnosis and Treatment of Thoracic Cancer, Zhejiang Cancer Hospital, 38 Guangji Road, Hangzhou, Zhejiang 310022, P.R. China

E-mail: xiaojiawangcn@126.com

${ }^{*}$ Contributed equally

Key words: breast cancer, cluster of differentiation 34, vascular endothelial growth factor, immunohistochemistry, clinicopathological factors for patients with breast cancer, a high level of CD34 expression may be a potential indicator of a poor prognosis.

\section{Introduction}

In recent years, a significant progression in the field of cancer research has been the clarification of the important role of tumor angiogenesis in the development of tumors and, thus, the significance of antiangiogenic therapy for the treatment of cancer $(1,2)$. Tumor angiogenesis refers to the process in which the growth of capillary vessels is induced by tumor cells and blood circulation is established in the tumor microenvironment. Angiogenesis is important in the growth, invasion and metastasis of the tumor. The concept of the angiogenic switch, which was initially proposed by Hanahan and Folkman (1) in 1996, further clarified that the proliferation and metastasis of primary solid tumors is dependent on angiogenesis, and is regulated by pro-angiogenesis factors and angiogenic inhibitory factors. Consequently, the association between capillary vessels, and tumor growth, invasion, metastasis and prognosis has become a popular research topic (2).

Cluster of differentiation (CD) 34 is a specific marker of vascular endothelial cells. In particular, CD34 is particularly sensitive to tumor angiogenesis, as it can clearly represent the state of neovascularization during the growth of a tumor (3). Vascular endothelial growth factor (VEGF), a factor involved in vascular endothelial proliferation, can specifically promote cell proliferation and angiogenesis, and is closely associated with the growth, invasion and metastasis of tumors. Therefore, CD34 and VEGF are two important indicators of tumor angiogenesis. Recently, the associations between CD34 and VEGF expression, and the clinicopathological characteristics of patients with cancer have been reported; however, the association between these associations and the survival of patients with cancer have rarely been reported. It has previously been demonstrated that the expression of CD34 and VEGF is of great importance for determining the prognosis of cancer patients (4-7), however, certain studies have argued that there is no significant association between the expression of CD34 and VEGF, and the survival of patients with cancer $(8,9)$. Therefore, the aim of the present study was to analyze the association between the expression of CD34 and 
VEGF, and the survival of patients with breast cancer, in order to identify the clinical significance of these two indicators in determining the prognosis of patients with breast cancer.

\section{Subjects and methods}

Subjects. Paraffin-embedded, formalin-fixed tissue blocks of resected breast cancer from 44 female patients (mean age, 57.14 years; range, 32-82 years) were obtained from the histopathology archives of Zhejiang Cancer Hospital (Hangzhou, China). All subjects were hospitalized and received treatment in Zhejiang Cancer Hospital between February 2006 and December 2006. Follow-up, which terminated in April 2013, was conducted for 41-88 months, with an average follow-up time of 62 months. Furthermore, no patients had received pre-operative radiotherapy or chemotherapy. A review of the clinicopathological data identified 1 case of medullary carcinoma, 1 case of metaplastic carcinoma (squamous cell carcinoma) and 42 cases of invasive ductal carcinoma. According to the 2010 edition of the Union for International Cancer Control (UICC) clinical staging system (10), there were 8 cases of stage I disease, 27 cases of stage II and 9 cases of stage III. If typed according to estrogen receptor (ER), progesterone receptor (PR) and human epidermal growth factor-2 (HER-2) immunohistochemical data, the tumors could be classified as luminal A-type (ER ${ }^{+}$ or $\mathrm{PR}^{+}$, plus HER-2), luminal $\mathrm{B}$-type $\left(\mathrm{ER}^{+}\right.$or $\mathrm{PR}^{+}$, plus HER-2 ${ }^{+}$), HER-2 overexpression-type (ER $/ \mathrm{PR}^{-} / \mathrm{HER}^{-} 2^{+}$) and triple-negative-type (ER/PR-/HER-2) breast cancer. Additionally, the p53 expression status was obtained from the clinicopathological data of the 44 specimens, among which there were 23 positive cases. The present study was conducted in accordance with the declaration of Helsinki and with the approval of the Ethics Committee of Zhejiang Cancer Hospital. Written informed consent was obtained from all participants.

Immunohistochemical analysis. The expression of CD34 and VEGF was detected using the EnVision immunohistochemical method (Dako, Glostrup, Denmark), according to the manufacturer's instructions. Briefly, the tissue sections were deparaffinized and rehydrated conventionally, and then incubated with $1 \mathrm{mM}$ EDTA for $10-15 \mathrm{~min}$ at $100^{\circ} \mathrm{C}$ in a pressure cooker for antigen retrieval. Following incubation with $3 \% \mathrm{H}_{2} \mathrm{O}_{2}$ for $5 \mathrm{~min}$ to remove endogenous peroxides, the sections were washed with phosphate-buffered saline and then incubated with rabbit monoclonal CD34 (clone EP373Y; dilution, 1:100; cat. no. ab81289; Abcam, Cambridge, MA, USA) or polyclonal VEGF (clone A-20; dilution, 1:300; cat. no. sc-152G; Santa Cruz Biotechnology, Inc., Dallas, TX, USA) antibodies for $2 \mathrm{~h}$ at room temperature, followed by incubation with horseradish peroxidase-conjugated secondary antibody (dilution, 1:100; cat. no. SA00001-17; Proteintech Group, Inc., Chicago, IL, USA). The signal was detected using 3,3'-diaminobenzidine substrate and then sections were counterstained with hematoxylin to visualize the nuclei.

\section{Interpretation of immunohistochemical results}

CD34 expression in vascular endothelial cells. According to the method described by Weidner (11), endothelial cell
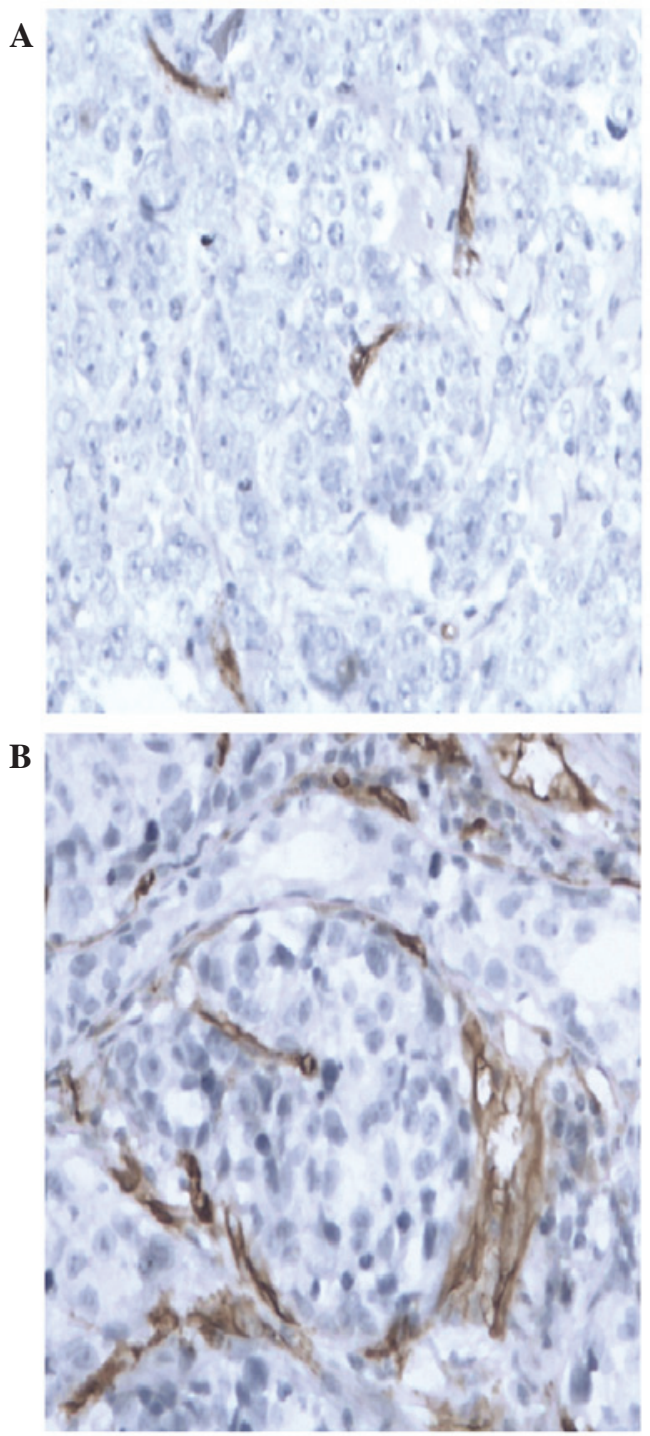

Figure 1. Expression of CD34 in breast cancer tissue: (A) low expression; (B) high expression. Magnification, x100.

clusters expressing CD34 and forming lumen or vessels were counted as individual microvessels. However, a luminal area larger than the sum of the diameters of eight erythrocytes, or a blood vessel with a thick muscular layer or a single positive cell were not counted as microvessels. Tumor sections were scanned under light microscopy (TH4-200; Olympus Corporation, Tokyo, Japan) at low power (magnification, $\mathrm{x} 40$ ) to identify vascular intensive areas (hot spots). Individual tumor microvessels were then counted at high power (magnification, x100) in five fields and the mean vessel count in three hot spots was used as the microvessel density (MVD). However, in contrast to the methods described by Keyhani et al (12), the present study defined a low expression CD34 level as an MVD value of $\leq 15 / \mathrm{HPF}$ (Fig. 1A) and a high CD34 expression level as an MVD value of $>15 /$ HPF (Fig. 1B).

VEGF expression in vascular endothelial cells. The expression intensity of VEGF and its distribution in the tumor samples were observed at high power (magnification, x100), and semi-quantitatively analyzed. Cytoplastic brown granules in the cytoplasm or membrane of the tumor cells were considered as positive for VEGF expression when the proportion of 
Table I. Association between CD34 and VEGF expression and various clinicopathological factors.

\begin{tabular}{|c|c|c|c|c|c|c|}
\hline \multirow[b]{2}{*}{ Parameter } & \multicolumn{2}{|c|}{ CD34 expression, $\mathrm{n}(\%)$} & \multirow[b]{2}{*}{ P-value } & \multicolumn{2}{|c|}{ VEGF expression, $\mathrm{n}(\%)$} & \multirow[b]{2}{*}{ P-value } \\
\hline & $\leq 15 / \mathrm{HPF}$ & $>15 / \mathrm{HPF}$ & & $0 /+$ & $++/+++$ & \\
\hline Age, years & & & 0.249 & & & 0.006 \\
\hline$\geq 50$ & $6(21.4)$ & $22(78.6)$ & & $6(21.4)$ & $22(78.6)$ & \\
\hline$<50$ & $6(37.5)$ & $10(62.5)$ & & $10(62.5)$ & $6(37.5)$ & \\
\hline TNM stage & & & 0.647 & & & 0.798 \\
\hline I-II & $9(25.7)$ & $26(74.3)$ & & $13(37.1)$ & $22(62.9)$ & \\
\hline III & $3(33.3)$ & $6(66.7)$ & & $3(33.3)$ & $6(66.7)$ & \\
\hline Vascular invasion & & & 0.873 & & & 0.018 \\
\hline Negative & $10(27.8)$ & $26(72.2)$ & & $16(44.4)$ & $20(55.6)$ & \\
\hline Positive & $2(25.0)$ & $6(75.0)$ & & $0(0)$ & $8(100)$ & \\
\hline Tumor size, $\mathrm{cm}$ & & & 1.000 & & & 1.000 \\
\hline$\leq 2$ & $3(27.3)$ & $8(72.7)$ & & $4(36.4)$ & $7(63.6)$ & \\
\hline$>2$ & $9(27.3)$ & $24(72.7)$ & & $12(36.4)$ & $21(63.6)$ & \\
\hline Lymph node metastasis & & & 0.255 & & & 0.907 \\
\hline Negative & $3(17.6)$ & $14(82.4)$ & & $6(35.3)$ & $11(64.7)$ & \\
\hline Positive & $9(33.3)$ & $18(66.7)$ & & $10(37.0)$ & $17(63.0)$ & \\
\hline p53 & & & 0.622 & & & 0.305 \\
\hline Negative & $5(23.8)$ & $16(76.2)$ & & $6(28.6)$ & $15(71.4)$ & \\
\hline Positive & $7(30.4)$ & $16(69.6)$ & & $10(43.5)$ & $13(56.5)$ & \\
\hline Molecular type & & & 0.214 & & & 0.186 \\
\hline Non-triple-negative & $5(20.0)$ & $20(80.0)$ & & $7(28.0)$ & $18(72.0)$ & \\
\hline Triple-negative & $7(36.8)$ & $12(63.2)$ & & $9(47.4)$ & $10(52.6)$ & \\
\hline ER & & & 0.658 & & & 0.160 \\
\hline Negative & $8(29.6)$ & $19(70.4)$ & & $12(44.4)$ & $15(55.5)$ & \\
\hline Positive & $4(23.5)$ & $13(76.5)$ & & $4(23.5)$ & $13(76.5)$ & \\
\hline PR & & & 0.736 & & & 0.851 \\
\hline Negative & $8(25.8)$ & $23(74.2)$ & & $11(35.5)$ & $20(64.5)$ & \\
\hline Positive & $4(30.8)$ & $9(69.2)$ & & $5(38.5)$ & $8(61.5)$ & \\
\hline HER-2 & & & 0.222 & & & 0.323 \\
\hline Negative & $11(31.4)$ & $24(68.6)$ & & $14(40.0)$ & $21(60.0)$ & \\
\hline Positive & $1(11.1)$ & $8(88.9)$ & & $2(22.2)$ & 7 (77.8) & \\
\hline
\end{tabular}

CD34, cluster of differentiation 34; VEGF, vascular endothelial growth factor; TNM, tumor-node-metastasis; ER, estrogen receptor; PR, progesterone receptor; HER2, human epidermal growth factor receptor-2.

immunoreactive cells was $\geq 5 \%$ [ $<5 \%$ staining, negative $(0)$; $5-25 \%$ staining, weakly positive $(+) ; 26-50 \%$ staining, positive $(++)$; and $>50 \%$ staining, strongly positive $(+++)$ ]. For statistical analysis, samples with $0 /+$ staining were included in the low VEGF expression group, while samples with $++/+++$ expression were included in the high VEGF expression group.

Statistical analysis. SPSS statistical software (version 17.0; SPSS, Inc., Chicago, IL, USA) was used to perform the statistical analyses in the present study. Comparisons between groups were analyzed using a $\chi^{2}$ test and univariate survival analysis was conducted using the Kaplan-Meier method. In addition, a log-rank test was performed to identify significant factors for Cox regression multivariate analysis and ultimately to determine independent factors affecting the survival of the patients. $\mathrm{P}<0.05$ was considered to indicate a statistically significant difference.

\section{Results}

Expression of CD34 in breast cancer. As determined by immunohistochemical analysis, 32 specimens of breast cancer expressed low levels of CD34, with an MVD of $\leq 15 / \mathrm{HPV}$, accounting for $72.7 \%$ of cases. By contrast, 12 specimens expressed high levels of CD34, with an MVD of $>15 / \mathrm{HPV}$, accounting for $27.3 \%$ of cases. The expression of CD34 had no significant correlation with the clinicopathological factors of the patients enrolled in the present study ( $\mathrm{P}>0.05$; Table I). However, Kaplan-Meier analysis demonstrated that the overall 
Table II. Cox multivariate analysis of 44 patients with breast cancer.

\begin{tabular}{|c|c|c|c|c|}
\hline Parameter & Risk ratio $(95 \% \mathrm{CI})$ & P-value & Adjusted risk ratio $(95 \% \mathrm{CI})$ & P-value \\
\hline CD34 (negative vs. positive) & $0.155(0.039-0.624)$ & $0.009^{\mathrm{a}}$ & $0.096(0.009-1.050)$ & 0.055 \\
\hline VEGF (negative vs. positive) & $0.471(0.098-2.270)$ & 0.348 & $0.497(0.041-6.108)$ & 0.585 \\
\hline TNM stage (I-II vs. III) & $0.091(0.022-0.372)$ & $0.001^{\mathrm{a}}$ & $0.023(0.002-0.322)$ & $0.005^{\mathrm{a}}$ \\
\hline $\begin{array}{l}\text { Molecular type (non-triple-negative } \\
\text { vs. triple-negative) }\end{array}$ & $0.563(0.151-2.098)$ & 0.392 & $0.021(0.001-0.330)$ & $0.006^{\mathrm{a}}$ \\
\hline Vascular invasion (negative vs. positive) & $0.802(0.166-3.869)$ & 0.784 & $0.476(0.026-8.644)$ & 0.616 \\
\hline Age $(\leq 50$ vs. $>50$ years $)$ & $0.196(0.025-1.570)$ & 0.125 & $0.007(0.000-0.643)$ & $0.032^{\mathrm{a}}$ \\
\hline
\end{tabular}

${ }^{\mathrm{a}} \mathrm{P}<0.05$. CI, confidence interval; CD34, cluster of differentiation 34; VEGF, vascular endothelial growth factor; TNM, tumor-node-metastasis .

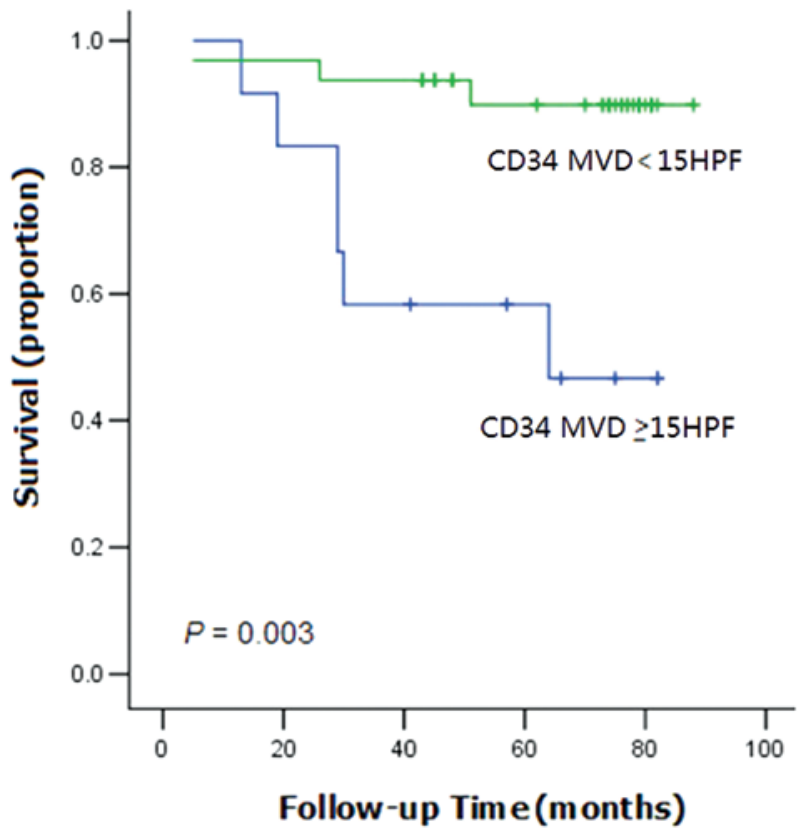

Figure 2. Survival curves of patients with breast cancer grouped by CD34 expression. CD34, cluster of differentiation 34; MVD, microvessel density.

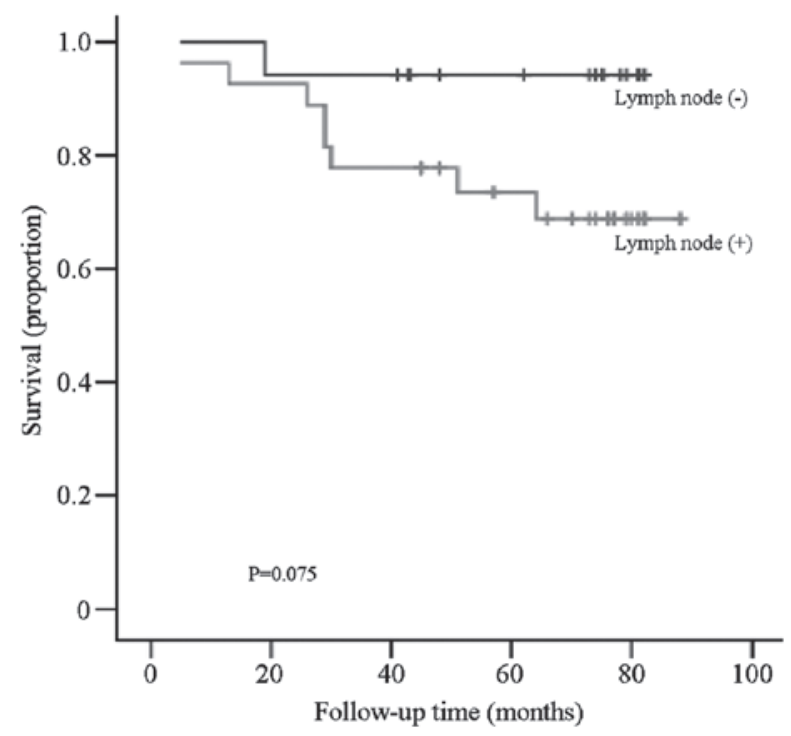

Figure 3. Survival curves of patients with and without lymph node metastasis.

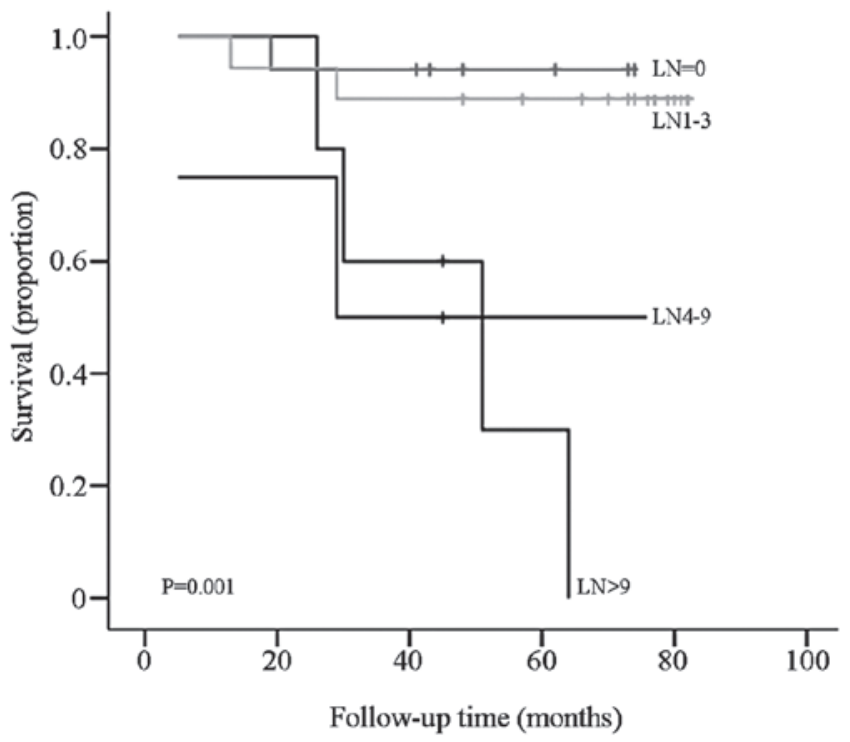

Figure 4. Survival curves of patients grouped by metastatic LN number. LN, lymph node.

survival (OS) time of the patients with high CD34 expression levels was significantly shorter than the OS time of patients with low CD34 expression ( $\mathrm{P}=0.003$; Fig. 2).

Expression of VEGF in breast cancer. Among the 44 cases of breast cancer tissue investigated, 16 cases (36.4\%) weakly expressed (0/+) VEGF and 28 cases (63.6\%) strongly expressed $(++/+++)$ VEGF. The OS of patients exhibiting high VEGF expression demonstrated no significant difference from that of the patients with low expression $(\mathrm{P}=0.366)$. When the tissues were divided into two groups by the patient's age ( $\geq 50$ and $<50$ years), the proportion of patients exhibiting high VEGF expression in the $\geq 50$ years group was significantly higher than that in the $<50$ years group ( 78.6 vs. $37.5 \%$; $\mathrm{P}=0.006)$. In terms of vessel infiltration, VEGF was highly expressed in all 8 patients with vessel infiltration (100\%), but was only expressed in 20/36 patients without vascular invasion (55.6\%); this difference was statistically significant $(\mathrm{P}=0.018)$ (Table I).

Cox regression multivariate analysis. Cox multivariate analysis identified that clinical stage, molecular type 
and age were independent prognostic factors for breas cancer $(\mathrm{P}=0.005, \mathrm{P}=0.006$ and $\mathrm{P}=0.032$, respectively), while the expression of CD34 was identified as a potential prognostic factor $(\mathrm{P}=0.055$; Table II $)$.

Association between the OS of patients and lymph node metastasis. There was no significant difference between the OS of breast cancer patients with and without lymph node metastasis ( $\mathrm{P}=0.075$; Fig. 3). However, when the samples were divided into four groups according to the metastatic lymph node number ( $\mathrm{N}, 0 ; \mathrm{N} 1,1-3 ; \mathrm{N} 2,4-9$; and N3, >10), the difference in OS between each group was statistically significant ( $\mathrm{P}=0.001$; Fig. 4).

\section{Discussion}

CD34, also known as human hematopoietic progenitor cell antigen, is a single-chain transmembrane glycoprotein with a molecular weight of 105-120 kDa, located on the long arm of chromosome 1. CD34 is an endothelial cell-specific marker, predominantly expressed on endothelial and hematopoietic progenitor cells, and closely associated with the process of angiogenesis (13). In addition, it is a more objective indicator for evaluating the extent of tumor angiogenesis. High expression of CD34 indicates a high MVD, i.e., more microvessels within the tumor tissue. Neovessels provide nutrients and oxygen for tumor cells and remove metabolic waste, promoting the growth rate of tumor (7). Furthermore, increased contact between the tumor cells and the blood vessels, the weak and thin neovessel wall, and the incomplete matrix membrane further promotes tumor cell adhesion and entrance of tumor cells into the vessel. Tumor cell metastasis can then occur via the blood circulation, allowing spread to other areas of the body and form new metastatic lesions. Therefore, an increase in the number of capillaries indicates an increased probability of invasion and metastasis (2). Ch'ng et al (14) analyzed CD34 expression in 94 invasive ductal breast cancer tissue samples and identified that CD34 was highly expressed in the youth group (aged $\leq 55$ years), but was not associated with other clinicopathological factors. In addition, the clinical stage of breast cancer was determined to be a major contributor to a poor prognosis. Similarly, Murri et al (15) reported the expression of CD34 in 168 patients with early invasive breast cancer. This study identified that an increased tumor MVD was correlated with the OS of the patients $(\mathrm{P}<0.05)$, and multivariate analysis identified albumin concentration, topical therapy, systemic therapy and tumor MVD as independent factors for predicting a poor prognosis $(\mathrm{P}<0.05)$. By contrast, Chuangsuwanich et al (16) used immunomarkers on tissue microarrays to classify the subtypes of breast cancer, and performed a correlation analysis between breast cancer subtype, and various clinicopathological features and prognostic markers, such as Ki-67 expression, p53 expression, MVD and VEGF expression. However, no significant elevation in MVD or VEGF expression was apparent. In the present study, the OS time of patients with high CD34 expression was significantly shorter compared with patients with low CD34 expression $(\mathrm{P}=0.003)$. Additionally, Cox multivariate analysis identified that CD34 expression at $>15 / \mathrm{HPV}$ was a potential indicator of a poor prognosis $(\mathrm{P}=0.055)$. Thus, detection of CD34 expression may provide a novel potential tumor marker for the clinical diagnosis and prognosis of patients with breast cancer.

VEGF is a highly specific endogenous vascular endothelial cell growth factor that activates tyrosine kinase receptors, predominantly via binding to its specific surface receptor, VEGF receptor-1 [VEGFR-1 (Flt-1)], and causing the initiation of signal conduction. This signaling can promote the mitosis of endothelial cells and eventually results in neovascularization. Furthermore, VEGF signaling can increase vascular permeability by increasing the production of enzymes required for the degradation of extracellular matrix (4). Good vascularization allows tumor cells to receive sufficient nutrients for rapid proliferation and to enter blood vessels for distant metastasis. The majority of previous studies have identified that VEGF expression is significantly increased in metastatic breast ductal carcinoma and is correlated with the poor clinical outcomes in patients with early-stage breast cancer $(12,17-19)$. Furthermore, VEGF may be an independent prognostic factor for the disease-free survival and OS of breast cancer patients (20). Thus, anti-VEGF therapy may have potential in the treatment of patients with breast cancer. Bevacizumab is a recombinant humanized monoclonal antibody for human VEGF that can prevent the biological effects of VEGF by neutralizing it. This neutralization of VEGF inhibits the formation of new blood vessels and reduces the oxygen supply, blood supply and nutrient supply to the tumor area, resulting in inhibited tumor growth (2,21). An international multi-center open randomized phase III clinical trial conducted by the Eastern Cooperative Oncology Group (E2100) and reported by Miller et al (21) appears to be the most representative trial of bevacizumab conducted thus far (22). In March 2008, bevacizumab in combination with paclitaxel was approved by the Food and Drug Administration (FDA) for use in the first-line treatment of metastatic breast cancer. However, a large number of clinical studies of bevacizumab were subsequently conducted and identified that, although improved progression-free survival was observed, improved OS was not (23-25); therefore, the application of bevacizumab for the treatment of metastatic breast cancer was revoked by the FDA. However, the administration of bevacizumab combined with paclitaxel for the treatment of metastatic breast cancer is still retained in the American National Comprehensive Cancer Network Guide (26). In addition, it is still widely applied in the treatment of colon cancer, lung cancer, glioblastoma, renal cell carcinoma and other tumors $(4,27)$.

Tumor angiogenesis is an important part of the process of breast cancer growth and metastasis; it is a complex multi-factorial and multi-step process that can be used to indicated if a tumor is malignant. In the process of tumor development, various angiogenic factors are preferentially expressed at different stages. However, as a key factor with the strongest pro-angiogenic activity, VEGF is expressed throughout the process of tumor development and is correlated with patient survival $(4,6)$. Ni et al $(28)$ reported that VEGF expression in 75 patients with breast cancer was negatively correlated with survival time, indicating that VEGF may be a malignant phenotype of breast cancer, with increased expression predicting a poor prognosis. By contrast, a study conducted 
by Dhakal et al (8) identified that VEGF expression is associated with the differentiation of squamous cell carcinoma of the vulva, but not with patient survival. Similarly, a previous study determined that VEGF expression is not associated with clinicopathological factors or survival in patients with colorectal cancer (9). Furthermore, a meta-analysis of 1,357 patients with breast cancer identified that overexpression of VEGF-C was not correlated with the survival of patients with breast cancer (29). In the present study, high VEGF expression was identified in all 8 cases of breast cancer with tumor thrombi. All the breast cancer tissues with vascular invasion highly expressed VEGF (100\%); this value was significantly higher than in the tissues not exhibiting vascular invasion $(55.6 \%$; $\mathrm{P}=0.018$ ). In addition, patients aged $\geq 50$ years presented with a higher VEGF expression rate (78.6\%) compared with patients aged $<50$ years $(37.5 \%)(\mathrm{P}=0.006)$. However, Kaplan-Meier analysis determined that differences in the OS of patients were not significant between the high and low VEGF expression groups $(\mathrm{P}=0.366)$. Thus, consistent with results reported by Gao et al (29), VEGF expression was not an independent prognostic factor for the breast cancer patients. However, data obtained in the present study identified that clinical stage, molecular typing and age were independent prognostic factors for patients with breast cancer $(\mathrm{P}=0.005, \mathrm{P}=0.006$ and $\mathrm{P}=0.032$, respectively), while the expression of CD34 was determined to be a potential independent prognostic factor.

Srabovic et al (30) examined VEGFR-1 and VEGF expressions levels in tumors and the adjacent tissue of 51 patients with breast cancer, and in the healthy breast tissue of 30 patients with benign breast diseases using immunohistochemical staining. The results demonstrated that the expression of VEGFR-1 and VEGF were significantly higher in the breast cancer tissues compared with the healthy breast tissues $(\mathrm{P}<0.01)$. Furthermore, a significant correlation was identified between VEGF and VEGFR-1 expression levels $(\mathrm{P}<0.05)$; while no significant correlation was observed between VEGF and VEGFR-1 expression, and tumor size, histological grade or hormone receptor status. Increased expression of VEGFR-1 and VEGF in breast cancer tissues, and significant correlation between the two proteins, indicates a possible role of the VEGF/VEGFR-1 signaling pathway in the development of breast cancer, although the potential prognostic value of VEGFR-1 has not yet been confirmed. A study conducted by Keyhani et al (12) demonstrated that angiogenic markers in breast cancer (for example, CD34) are potentially useful tools for determining the most appropriate priority setting for delivering antiangiogenic agents.

Although the sample size used in the present study was small, the OS of patients according to the number of metastatic lymph nodes (staged according to the 2010 edition of the UICC staging system) demonstrated statistically significant differences. Therefore, the current findings provide a greater scientific basis for employing the UICC staging system in breast cancer.

\section{Acknowledgements}

The present study was supported by a grant from the Medical Science and Technology Development Program of Zhejiang Province (grant no. 2010KYB020).

\section{References}

1. Hanahan D and Folkman J: Patterns and emerging mechanisms of the angiogenic switch during tumorigenesis. Cell 86: 353-364, 1996.

2. Ziyad S and Iruela-Arispe ML: Molecular mechanisms of tumor angiogenesis. Genes Cancer 2: 1085-1096, 2011.

3. Cao Y, Zhang ZL, Zhou M, et al: Pericyte coverage of differentiated vessels inside tumor vasculature is an independent unfavorable prognostic factor for patients with clear cell renal cell carcinoma. Cancer 119: 313-324, 2013.

4. Zorgetto VA, Silveira GG, Oliveira-Costa JP, Soave DF, Soares FA and Ribeiro-Silva A: The relationship between lymphatic vascular density and vascular endothelial growth factor A (VEGF-A) expression with clinical-pathological features and survival in pancreatic adenocarcinomas. Diagn Pathol 8: 170, 2013.

5. Horn LC, Schreiter C, Canzler A, Leonhardt K, Einenkel J and Hentschel B: CD34(low) and SMA(high) represent stromal signature in uterine cervical cancer and are markers for peritumoral stromal remodeling. Ann Diagn Pathol 17: 531-535, 2013.

6. Zhan H, Liang H, Liu X, Deng J, Wang B and Hao X: Expression of Rac1, HIF-1 $\alpha$ and VEGF in gastric carcinoma: correlation with angiogenesis and prognosis. Onkologie 36: 102-107, 2013.

7. Sundov Z, Tomic S, Alfirevic S, et al: Prognostic value of MVD, LVD and vascular invasion in lymph node-negative colon cancer. Hepatogastroenterology 60: 432-438, 2013.

8. Dhakal HP, Nesland JM, Førsund M, Trope CG and Holm R: Primary tumor vascularity, HIF-1 $\alpha$ and VEGF expression in vulvar squamous cell carcinomas: their relationships with clinicopathological characteristics and prognostic impact. BMC Cancer 13: 506, 2013.

9. Anannamcharoen S and Nimmanon T: Study of the vascular endothelial growth factor (VEGF) expression and microvascular density (MVD) in primary colorectal cancer specimens. J Med Assoc Thai 95: 1041-1047, 2012.

10. Edge SB, Byrd DR, Compton CC, Fritz AG, Greene FL and Trotti A (eds): Breast Cancer. In: AJCC Cancer Staging Manual. 7th edition. Springer-Verlag, New York, NY, pp223-240, 2009.

11. Weidner N: Current pathologic methods for measuring intratumoral microvessel density within breast carcinoma and other solid tumors. Breast Cancer Res Treat 36: 169-180, 1995.

12. Keyhani E, Muhammadnejad A, Behjati F, et al: Angiogenesis markers in breast cancer - potentially useful tools for priority setting of anti-angiogenic agents. Asian Pac J Cancer Prev 14: 7651-7656, 2013.

13. Satterthwaite AB, Burn TC, Le Beau MM and Tenen DG: Structure of the gene encoding CD34, a human hematopoietic stem cell antigen. Genomics 12: 788-794, 1992.

14. Ch'ng ES, Tuan Sharif SE and Jaafar H: Characteristics of invasive breast ductal carcinoma, NOS, diagnosed in a tertiary institution in the East Coast of Malaysia with a focus on tumor angiogenesis. Asian Pac J Cancer Prev 13: 4445-4452, 2012.

15. Murri AM, Hilmy M, Bell J, et al: The relationship between the systemic inflammatory response, tumour proliferative activity, T-lymphocytic and macrophage infiltration, microvessel density and survival in patients with primary operable breast cancer. $\mathrm{Br} \mathbf{J}$ Cancer 99: 1013-1019, 2008.

16. Chuangsuwanich T, Pongpruttipan T, O-Charoenrat P, Komoltri C, Watcharahirun S and Sa-Nguanraksa D: Clinicopathologic features of breast carcinomas classified by biomarkers and correlation with microvessel density and VEGF expression: a study from Thailand. Asian Pac J Cancer Prev 15: 1187-1192, 2014.

17. Staton CA, Hoh L, Baldwin A, Shaw L, Globe J, Cross SS, Reed MW and Brown NJ: Angiopoietins 1 and 2 and Tie-2 receptor expression in human ductal breast disease. Histopathology 59: 256-263, 2011.

18. Davidson B, Stavnes HT, Førsund M, Berner A and Staff AC: CD105 (Endoglin) expression in breast carcinoma effusions is a marker of poor survival. Breast 19: 493-498, 2010.

19. Zhao YC, Ni XJ, Li Y, Dai M, Yuan ZX, Zhu YY and Luo CY: Peritumoral lymphangiogenesis induced by vascular endothelial growth factor $\mathrm{C}$ and $\mathrm{D}$ promotes lymph node metastasis in breast cancer patients. World J Surg Oncol 10: 165, 2012.

20. Acs G, Paragh G, Rakosy Z, et al: The extent of retraction clefts correlates with lymphatic vessel density and VEGF-C expression and predicts nodal metastasis and poor prognosis in early-stage breast carcinoma. Mod Pathol 25:163-177, 2012.

21. Miller K, Wang M, Gralow J, et al: Paclitaxel plus bevacizumab versus paclitaxel alone for metastatic breast cancer. $\mathrm{N}$ Engl $\mathrm{J}$ Med 357: 2666-2676, 2007. 
22. Miles DW, Chan A, Dirix LY, et al: Phase III study of bevacizumab plus docetaxel compared with placebo plus docetaxel for the first-line treatment of human epidermal growth factor receptor 2-negative metastatic breast cancer. J Clin Oncol 28: $3239-3247,2010$.

23. Robert NJ, Diéras V, Glaspy J, et al: RIBBON-1: randomized, double-blind, placebo-controlled, phase III trial of chemotherapy with or without bevacizumab for first-line treatment of human epidermal growth factor receptor 2-negative, locally recurrent or metastatic breast cancer. J Clin Oncol 29: 1252-1260, 2011.

24. Gonçalves A, Deblock M, Esterni B, et al: Docetaxel first-line therapy in HER2-negative advanced breast cancer: a cohort study in patients with prospectively determined HER 2 status. Anticancer Drugs 20: 946-952, 2009.

25. Brufsky AM, Hurvitz S, Perez E, Swamy R, Valero V, O'Neill V and Rugo HS: RIBBON-2: a randomized, double-blind, placebocontrolled, phase III trial evaluating the efficacy and safety of bevacizumab in combination with chemotherapy for second-line treatment of human epidermal growth factor receptor 2-negative metastatic breast cancer. J Clin Oncol 29: 4286-4293, 2011.
26. Theriault RL, Carlson RW, Allred C, et al: National Comprehensive Cancer Network: Breast cancer, version 3.2013: Featured updates to the NCCN guidelines. J Natl Compr Canc Netw 11: 753-760, 2013.

27. Huang H, Zheng Y, Zhu J, Zhang J, Chen H and Chen X: An updated meta-analysis of fatal adverse events caused by bevacizumab therapy in cancer patients. PLoS One 9: e89960, 2014.

28. Ni X, Zhao Y, Ma J, Xia T, Liu X, Ding Q, Zha X and Wang S: Hypoxia-induced factor-1 alpha upregulates vascular endothelial growth factor $\mathrm{C}$ to promote lymphangiogenesis and angiogenesis in breast cancer patients. J Biomed Res 27: 478-485, 2013.

29. Gao S, Ma JJ and Lu C: Prognostic significance of VEGF-C immunohistochemical expression in breast cancer: a meta-analysis. Tumour Biol 35: 1523-1529, 2014.

30. Srabovic N, Mujagic Z, Mujanovic-Mustedanagic J, Softic A, Muminovic Z, Rifatbegovic A and Begic L: Vascular endothelial growth factor receptor-1 expression in breast cancer and its correlation to vascular endothelial growth factor $\mathrm{a}$. Int $\mathrm{J}$ Breast Cancer 2013: 746749, 2013. 